\title{
MICOPLASMI UROGENITALI: FREQUENZA DI ISOLAMENTO E SENSIBILITÀ AGLI ANTIBIOTICI. CINQUE ANNI DI OSSERVAZIONE.
}

Vincenti A., Greco F.

Laboratorio di Analisi Chimico-cliniche e Microbiologia

"Data Medica", via Enrico Toti 5,

51016 Montecatini Terme (PT)

\section{Introduzione}

Nei casi di affezione del tratto uro-genitale sono isolate soprattutto due specie di Micoplasmi, Ureaplasma urealyticum (U.u.) e Mycoplasma hominis (M.h.). Nel nostro studio sono stati considerati gli esami effettuati per la ricerca dei Micoplasmi nel periodo da Gennaio 2001 ad Aprile 2005 per valutare la prevalenza ed eventuali variazioni nell'attività antimicrobica.

\section{Metodi}

E' stato utilizzato il kit della ditta bioMerieux, in particolare nel 2001 e nel 2002 il kit Mycoplasma IST con sei antibiotici (doxiciclina, josamicina, ofloxacina, eritromicina, tetraciclina e pristinamicina), dal 2003 il Mycoplasma IST 2 con l'aggiunta di tre antibiotici (ciprofloxacina, azitromicina e claritromicina). I kit prevedono l'uso di un brodo di coltura selettivo per i micoplasmi da inoculare in una galleria per l'identificazione, la conta e la sensibilità verso gli antibiotici.

\section{Risultati}

Lo studio è stato condotto su 590 campioni provenienti da tamponi endocervicali (TE) e uretrali (TU), da urina (U) e da sperma (SP). 195 sono risultati positivi alla coltura, circa il $33 \%$. U. urealiticum è stato il microrganismo più frequentemente isolato, $67 \%$ (Tabella 1)

Tabella: U.urealyticum e/o M.hominis isolati dalle varie sedi di prelievo

\begin{tabular}{lcccccc}
\hline & T.E. & U. & SP. & T.U. & TOT & \% \\
\hline U.u. & 88 & 26 & 9 & 7 & 130 & $\mathbf{6 7}$ \\
\hline M. h. & 31 & 0 & 0 & 5 & 36 & $\mathbf{1 8}$ \\
\hline U.u.+M.h. & 15 & 9 & 3 & 2 & 29 & $\mathbf{1 5}$ \\
\hline & $\mathbf{1 3 4}$ & $\mathbf{3 5}$ & $\mathbf{1 2}$ & $\mathbf{1 4}$ & $\mathbf{1 9 5}$ & \\
\hline
\end{tabular}

\section{Conclusioni}

Il corso dei cinque anni non si sono osservate variazioni significative nel trend di sensibilità agli antibiotici saggiati. Per U.urealyticum non si è avuto nessun ceppo resistente a doxiciclina, josamicina e pristinamicina, il $7 \%$ resistente ad eritromicina e tetraciclina, circa il $30 \%$ resistente ai fluorochinolonici, il $4 \%$ resistente ad azitromicina e il $2 \%$ resistente a claritromicina. Per M.hominis tutti i ceppi sono risultati sensibili a josamicina e pristinamicina e resistenti ai macrolidi, 1' $85 \%$ sensibile a doxiciclina, 1'80\% sensibile a tetraciclina, circa il $72 \%$ sensibile ai fluorochinolonici. 